\title{
Tonsillectomy discharge information - An improvement in both patient safety and satisfaction
}

\author{
Alison Carter, Elinor Warner, Andrew Roberton, Xiaxin Liu, Ayeshah Abdul-Hamid, Ravinder Natt, Claire Hopkins \\ Guy's and St. Thomas' Hospital
}

\begin{abstract}
Approximately 20,000 adult and 25,000 paediatric tonsillectomies are performed each year in England. $0.9 \%$ of these patients return to theatre for post-tonsillectomy bleeding. The Royal College of Surgeons of England (RCSEng) have produced guidelines regarding emergency surgery, with standards for tonsillectomy discharge information. We audited our compliance with these guidelines and patient satisfaction regarding the information currently provided.
\end{abstract}

Theatre records identified all tonsillectomies carried out between December 2012 and February 2013. 71 patients and their electronic discharge information were reviewed for post-operative bleeding information. Each patient was contacted, with a second call made to those who did not answer. 35 patients took part in our telephone questionnaire. Only $35 \%$ of patients had post-operative bleeding information on their discharge summary. $51 \%$ received no written information either in clinic or on the day of surgery, $66 \%$ recalled a verbal explanation. Only $54 \%$ knew to go to A\&E if they experienced bleeding. $40 \%$ were not satisfied with their discharge information, stating that they wanted to know about bleeding, recovery expectations, and information regarding oral intake.

A focus group was formed to discuss potential solutions to the audit outcomes and a tonsillectomy leaflet was produced inline with the trust patient information template. It contained specific instructions regarding bleeding and the nearest A\&E contact details. It was reviewed by three tonsillectomy patients and their feedback regarding further information on post-operative diet, throat appearance and pain expectations was incorporated. A second cycle of the audit took place between August and September 2013. Results showed improvement, with $83 \%$ receiving an information leaflet and $100 \%$ a verbal explanation. $100 \%$ of patients were satisfied with their discharge information and $100 \%$ knew what to do if they bled. As a result we now meet the standards set out by the RCSEng and have increased our patient safety and satisfaction rates.

\section{Problem}

Patients undergoing tonsillectomies often have a limited amount of contact with an otolaryngologist. Once referred by their GP, they are likely to have one initial consultation prior to being listed for surgery. They are admitted on the day of their tonsillectomy and are often discharged the same day. This provides a limited amount of time in which the patient is able to both process the information given to them, as well as clarify any aspects of the procedure they are unclear about. As clinicians, with busy clinics and time pressures, we can also be guilty of under-explaining procedures and not giving enough space for the patient to ask any questions they might have.

A key safety aspect of tonsillectomy discharge information regards what to do if the patient has bleeding post operatively. Postoperative bleeding can in the extremes be fatal, and therefore the importance of initial management by the patient should not be underestimated. Prompt presentation to an accident and emergency department with the facilities to deal with haemorrhage is vital.

\section{Background}

In England, tonsillectomies are one of the most common surgical procedures, accounting for approximately 20,000 adult and 25,000 paediatric operations per year. Of these patients, $0.9 \%$ return to the operating theatre due to post-tonsillectomy haemorrhage (1). In 2011, the Royal College of Surgeons of England (RCSEng) produced the guidance 'Emergency Surgery - Standards for unscheduled surgical care' (2). As well as general guidance, the document contains specific specialty related guidance. It states "post tonsillectomy discharge information specifies contact details for the patient's nearest centre. Centre requires skills and equipment to deal with arrest of haemorrhage, including blood transfusion capability, immediate theatre access and ageappropriate anaesthetist out of hours".

At our institution, the current written discharge information given out to patients did not include the contact details of their nearest A\&E. We were also unable to account for if patients were being told the importance of post-tonsillectomy bleed management verbally, or if indeed patients were satisfied with the discharge information given in general.

\section{Baseline Measurement}

Theatre records were used to identify all tonsillectomies carried out between December 2012 and February 2013. 71 patients and their 
electronic discharge information were reviewed for information about bleeding post-operatively. Each patient was called from a hospital telephone, with a second call made to those who did not answer. 35 patients agreed to take part in our telephone questionnaire. Only $35 \%$ of patients had information related to postoperative bleeding on their discharge summary. $51 \%$ received no written information about their procedure either in clinic or on the day of surgery, $66 \%$ recalled receiving a verbal explanation. Only $54 \%$ would have known to go to $A \& E$ if they experienced bleeding. $40 \%$ were not satisfied with their discharge information, stating that they wanted to know what to do if they had bleeding, what to expect during their recovery, and information regarding oral intake.

\section{Design}

A focus group was formed to discuss potential solutions to the audit outcomes. With regards to improving discharge information, it was decided that written information should be improved as it was easier to regulate and standardise, but also gave the patient the opportunity to use it as an ongoing resource once discharged. Written information was given to the patient in two forms - the electronic discharge information (which was also issued to their GP) and a tonsillectomy discharge information leaflet. It was discussed amongst the focus group that the electronic discharge information was difficult to regulate given the high turnover of surgeons which rotate through from different hospitals during their training, and would require numerous education sessions for new trainees. A new tonsillectomy discharge information leaflet provided a better and more achievable intervention. There was also currently no institution-specific leaflet in circulation for tonsillectomies, but other surgical departments in our institution had had success producing leaflets for their procedures inline with the trust patient information leaflet template.

\section{Strategy}

A new institution specific tonsillectomy leaflet was produced and went through various cycles of review. Cycle 1 consisted of review by our supervising consultant. Cycle 2 consisted of review by the directorate lead and the trust patient information lead. During this cycle the leaflet was standardised in format to match those already in circulation, and also included cross references to other leaflets already produced by the trust. Cycle 3 consisted of review by three post-operative tonsillectomy patients. Their feedback highlighted some areas where the wording needed to be changed due to being too medical, for example 'tonsil bed' was changed to 'throat'. It also raised a desire for patients to have more information regarding postoperative diet, pain expectations, and what to expect in terms of post-operative appearance. After these changes were made, the leaflet was re-reviewed by the directorate lead and the trust patient information lead and was published on the trust website as well as printed for distribution to each tonsillectomy patient.

\section{Results}

Post-intervention, the audit was repeated during a four week period in August and September 2013. 14 patients and their electronic discharge information were reviewed for information about bleeding post-operatively. Each patient was called from a hospital telephone, with a second call made to those who did not answer. 12 patients agreed to take part in our telephone questionnaire. $83 \%$ compared to the previous $49 \%$ had received an information leaflet. $100 \%$ compared to the previous $71 \%$ had received a verbal explanation. $100 \%$ compared to the previous $60 \%$ were satisfied with their discharge information. 100\% compared to the previous $63 \%$ knew what to do if they bled, and $100 \%$ compared to the previous $0 \%$ specified the contact details of the A\&E department and therefore met the RCSEng Emergency Surgery Guidelines.

\section{Lessons and Limitations}

Lessons learned included the importance of a multi-disciplinary review of the leaflet. Junior doctors, consultants, patients and patient information lead managers each had vital contributions to the final version of the leaflet, and were all different to what other members of the team had commented on.

Challenges included promoting the new patient information leaflet, and raising awareness within the day surgery department. Now that it is in regular use, we anticipate it to be a sustainable intervention. A further cycle of the audit could confirm whether this has been the case and allow further promotion if necessary. Regarding cost effectiveness, the leaflet is easily printed from the trust intranet so involves simple A4 sheet printing costs onto the recycled paper currently used in the hospital printers. Patients also have the option of downloading the leaflet from the trust internet site at no cost to the hospital.

\section{Conclusion}

Creating an institution-specific tonsillectomy patient information leaflet has not only resulted in meeting the Royal College of Surgeons of England's Emergency Surgery Guidelines, but has also improved patient safety in the event of post-tonsillectomy bleeding, and has also increased patient satisfaction. In the future this could be re-audited to ensure we continue to meet these standards and satisfaction rates, and information leaflets could be produced for other key procedures within our department.

\section{References}

1. Clinical Effectiveness Unit. National Prospective Tonsillectomy Audit. The Royal College of Surgeons of England. 2005.

2. Professional Standards and Regulations Department. Emergency Surgery - Standards for unscheduled surgical care. The Royal College of Surgeons of England. 2011.

\section{Declaration of interests}

None declared

\section{Acknowledgements}




\section{BMJ Quality Improvement Reports}

Guy's and St. Thomas' Hospital Patient Information Leads. 